\title{
Investigation of ischemia modified albumin, oxidant and antioxidant markers in acute myocardial infarction
}

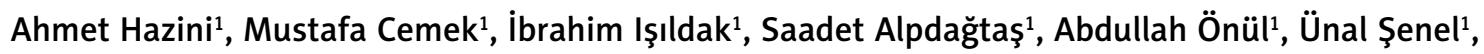 \\ Tuba Kocaman ${ }^{1}$, Ali Dur ${ }^{2}$, Mustafa Iraz ${ }^{3}$, Hüseyin Uyarel ${ }^{4}$ \\ ${ }^{1}$ Department of Bioengineering (Biochemistry Division), Chemical and Metallurgical Engineering Faculty, Yıldız Technical University, \\ Istanbul, Turkey \\ ${ }^{2}$ Department of Emergency, Faculty of Medicine, Bezmialem Vakıf University, Istanbul, Turkey \\ ${ }^{3}$ Department of Pharmacology, Faculty of Medicine, Istanbul Medeniyet University, Istanbul, Turkey \\ ${ }^{4}$ Department of Cardiology, Faculty of Medicine, Bezmialem Vakif University, Istanbul, Turkey
}

Postep Kardiol Inter 2015; 11, 4 (42): 298-303

DOI: $10.5114 /$ pwki.2015.55600

\begin{abstract}
A bstract
Introduction: Acute myocardial infarction (AMI) is still one of the most common causes of death worldwide. In recent years, for diagnosis of myocardial ischemia, a new parameter, called ischemia modified albumin (IMA), which is thought to be more advantageous than common methods, has been researched.

Aim: In this study, systematic analysis of parameters considered to be related to myocardial ischemia has been performed, comparing between control and myocardial ischemia groups.

Material and methods: We selected 40 patients with $\mathrm{AMI}$ and 25 healthy controls for this study. Ischemia modified albumin levels, glutathione peroxidase (GPx), superoxide dismutase (SOD), and catalase (CAT) antioxidant enzyme activities and non-enzymatic antioxidants such as retinol, $\alpha$-tocopherol, $\beta$-carotene and ascorbic acid levels were investigated in both groups. Glutathione (GSH) and malondialdehyde (MDA) levels, which are indicators of oxidative stress, were compared between patient and control groups.

Results: Ischemia modified albumin levels were found significantly higher in the AMI diagnosed group when compared with controls. The MDA level was elevated in the patient group, whereas the GSH level was decreased. SOD, GPx and CAT enzyme levels were decreased in the patient group, where it could be presumed that oxidative stress causes the cardiovascular diseases.

Conclusions: Due to the increased oxidative stress, non-enzymatic and enzymatic antioxidant capacity was affected. Systematic investigation of parameters related to myocardial infarction has been performed, and it is believed that such parameters can contribute to protection and early diagnosis of AMI and understanding the mechanism of development of the disease.
\end{abstract}

Key words: myocardial infarction, ischemia modified albumin, oxidative stress, enzymatic and non-enzymatic antioxidants.

\section{Introduction}

Recently, research has revealed that cardiovascular disease (CVD) is coming to have an increasing role as the main cause of mortality and morbidity worldwide. Studies indicate that death rates from CVD in the entire world between 1990 and 2020 will have risen from 28.9\% to $36.3 \%$ [1]. Atherosclerosis itself plays a role in more than half of all deaths in the world [2]. Atherosclerosis, which is one of the most important causes of myocardial infarction, is a kind of vessel disease, characterized by loss of elasticity and thickness of the arteries, especially coronary and cerebral arteries. Many hypotheses about the causes of atherosclerosis have been proposed. In the light of research it has been demonstrated that the most consistent one is the oxidative stress hypothesis $[3,4]$.

In the case of inability in antioxidant defense system, reactive oxygen species (ROS) accumulates in the body due to various reasons. In general, this situation is called oxidative stress $[5,6]$. Free radicals interact with biomolecules due to their reactive structure. Thus, oxidative stress was reported to be related to cancer, cardiovascular disease and progression of the aging process [7].

Antioxidants inhibit cell damage related to oxidation, by preventing the formation of active oxygen or by binding to formed active oxygen [8]. Antioxidants are classified according to molecular structure as enzymatic (superoxide

\section{Corresponding author:}

Prof. Dr Mustafa Cemek, Department of Bioengineering, Biochemistry Division, Chemical and Metallurgical Engineering Faculty, Yıldız Technical University, Istanbul, Turkey, phone: +90 212383 4632, fax: +90 212383 4625, e-mail: mcemek@yahoo.com Received: 7.07.2014, accepted: 20.04.2015. 
dismutase - SOD, glutathione peroxidase - GPx, catalase - CAT) and non-enzymatic (glutathione - GSH, $\alpha$-tocopherol, $\beta$-carotene, retinol). Additionally they are classified as intracellular (SOD, CAT, GPx), extracellular (albumin, vitamins C) and membrane antioxidants ( $\alpha$-tocopherol, retinol) depending on their presence in the cell [9].

Ischemia is a restriction in blood supply to tissues, causing a shortage of oxygen needed for cellular metabolism. Ischemia is generally caused by arterial spasm or blockage of the arterial flow. The most common and life-threatening forms of ischemia are cardiac ischemia and cerebral ischemia. Acute myocardial infarction (AMI) is still one of the most common causes of death worldwide. All symptoms and signs which show up due to AMI are known as coronary syndrome [1, 2].

In the diagnosis of myocardial ischemia together with ECG findings, biochemical tests are usually used by determining creatine kinase $M B(C K-M B)$ and troponin levels. However, these tests are inadequate for diagnosis in some cases, especially in the early stage of these attacks. In recent years, for the diagnosis of myocardial ischemia a new parameter has been researched, called ischemia modified albumin (IMA), which is thought to be more advantageous than common biochemical methods. In previous researches, it was shown that formation of IMA starts immediately following the tissue ischemia and returns to normal levels within $48 \mathrm{~h}$. The most important reason for the formation of IMA is thought to be that free radicals are produced in the ischemic tissue.

An early and accurate diagnosis of AMI remains one of the most difficult problems facing emergency department clinicians. Therefore, in this study, systematic analysis of parameters that are considered to be related to AMI was performed in the study groups.

\section{Aim}

For this purpose, IMA levels, cardiac and oxidative stress markers, and enzymatic and non-enzymatic antioxidants were investigated in patient and controls groups.

\section{Material and methods}

\section{Chemicals}

Potassium phosphate, sodium phosphate dibasic dihydrate, hydrogen peroxide, ethanol, sodium chloride, nitric acid, perchloric acid, phosphate buffer, GSH, thiobarbituric acid, EDTA, 5,5-dithiobis-(2-nitrobenzoic acid), disodium hydrogen phosphate, hexane, sodium nitrite, sodium nitrate, albumin, and dithiothreitol were purchased from Sigma Aldrich (USA). Commercial kits of GPX, CAT, and SOD were obtained from Cayman Chemical (USA). All other chemicals and reagents used in this study were of analytical grade. Ultra-distilled water was used as the solvent.

\section{Patients}

The present study was approved by the Human Ethical Committee, Bezmialem Vakıf University, Faculty of Medicine, Istanbul, Turkey. The study group consisted of 40 patients diagnosed with acute myocardial infarction at Bezmialem Vakif University, Emergency Department. The control group included 25 healthy volunteers without any diagnosed heart problem. For the diagnosis of $A M I$, typical chest pain suggestive of acute coronary syndrome, ischemic ECG findings and CK-MB elevation were assessed. In the control group, without considering risk factors, healthy individuals who have no any heart disease were included.

Patients who reported a longer than 24-hour delay from symptom onset to emergency department admission were excluded. For the diagnosis of AMI one of the following criteria was searched. Chest pain, ST-segment elevation more than $1 \mathrm{~mm}$ and increased cardiac markers. Patients with thyroid and renal disease, chronic inflammatory disease and undergoing surgery within the last year were excluded from study group. All patients between 30 and 80 years old suffering from myocardial infarction were included. Patients with non-ischemic heart disease were also excluded from the study [10].

\section{Biochemical analysis}

Whole blood samples were collected into heparinized tubes and whole blood MDA and GSH levels were studied on the day of admission. The serum and erythrocyte samples were stored in a polystyrene plastic tube at $-70^{\circ} \mathrm{C}$ until the time of analysis. Serum ascorbic acid, retinol and $\alpha$-tocopherol activities were studied using a spectrophotometer (Shimadzu U.V. Visible 1601).

The MDA (as an important indicator of oxidative stress) levels were measured according to the method of Jain et al. based on spectrophotometry [11]. Whole blood GSH, $\beta$-carotene and vitamin A (retinol) concentrations were measured by the spectrophotometric method [12, 13]. Vitamin E ( $\alpha$-tocopherol) was analyzed colorimetrically [14]. Serum vitamin C (ascorbic acid) level was determined after derivatization with 2,4-dinitrophenylhydrazine [15].

Serum vitamin C (ascorbic acid) level was determined after derivatization with 2,4-dinitrophenylhydrazine [16].

Catalase, SOD and GPx activities were determined by using Cayman's ELISA kit according to the manufacturer's instructions (Cayman Chemical, USA) [17-19].

For determination of IMA level, the albumin cobalt binding test was used. The test developed by Bar-Or et al. was based on measuring descending albumin cobalt binding capacity [20]. The result of absorbance was reported as absorbance units (ABSU).

\section{Statistical analysis}

The results were expressed as mean SD. Student's $t$-test was used to compare the mean values of differ- 
ent biochemical parameters between $\mathrm{AMI}$ and control groups. In all data analysis, a value of $p<0.05$ was considered statistically significant.

\section{Results}

Results of biochemical analyses of $\mathrm{AMI}$ patient and control groups are presented in Figures $1-3$. Mean values of patient serum IMA (0.527 \pm 0.08$)$, cTnI (0.614 \pm 0.59$)$ and CK-MB (22.875 \pm 13.23$)$ levels were significantly

A

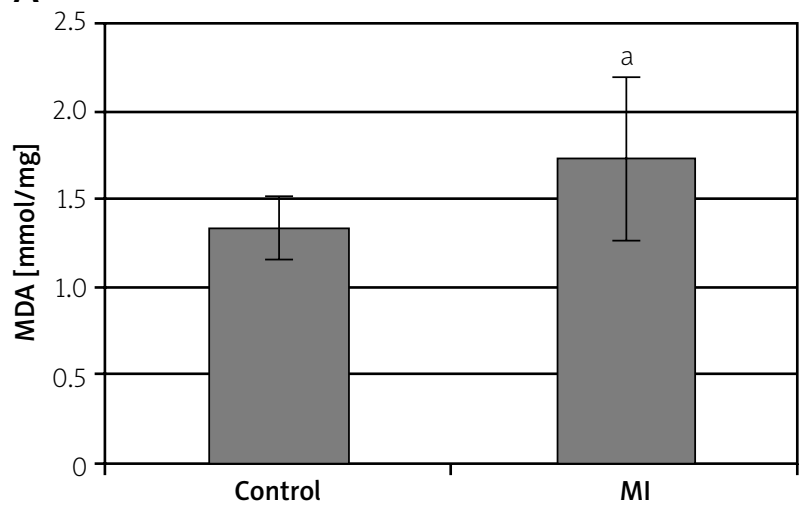

higher than in the control group $(0.347 \pm 0.05,0.027$ $\pm 0.01,6.54 \pm 1.52$, respectively) ( $p<0.001)$. Control group erythrocyte SOD $(0.157 \pm 0.02)$, CAT $(17.595 \pm 2.63)$ and GPx (58.655 \pm 7.41$)$ antioxidant enzyme activity levels compared to the patient group were markedly lower $(0.178 \pm 0.01,20.136 \pm 3.12$ and $64.691 \pm 5.94)(p<0.01, p<$ 0.001 and $p<0.01$ ) respectively (Figure 1 ).

While whole blood MDA control group levels (1.335 \pm 0.18 ) were lower than AMI patient group levels (1.735

B

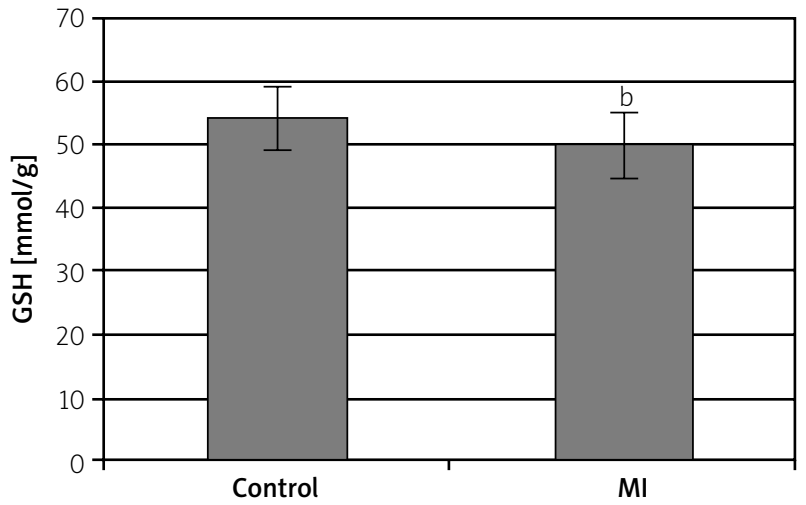

Figure 1. Oxidant and non-enzymatic antioxidant levels for each group. A - Whole blood MDA levels. B - Whole blood GSH levels

All values are expressed as mean $\pm S D:^{a} p<0.01$ with respect to control; ${ }^{b} p<0.001$ with respect to control. MDA - Malondialdehyde, GSH - reduced glutathione.

A

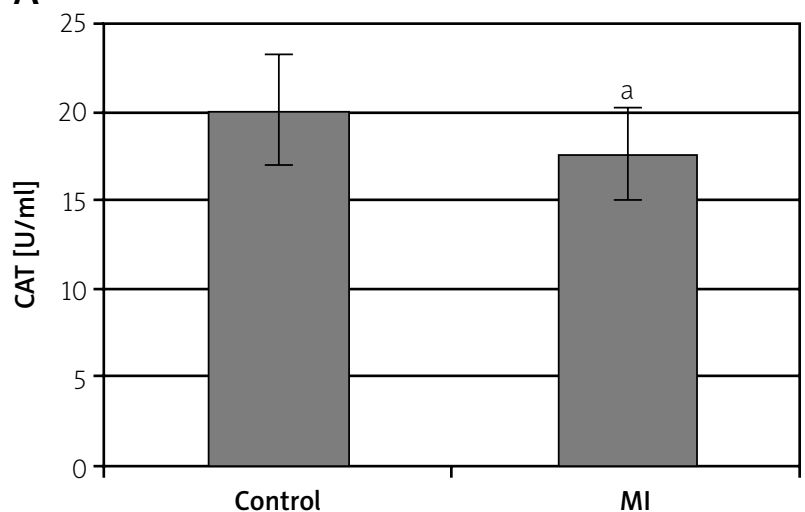

C

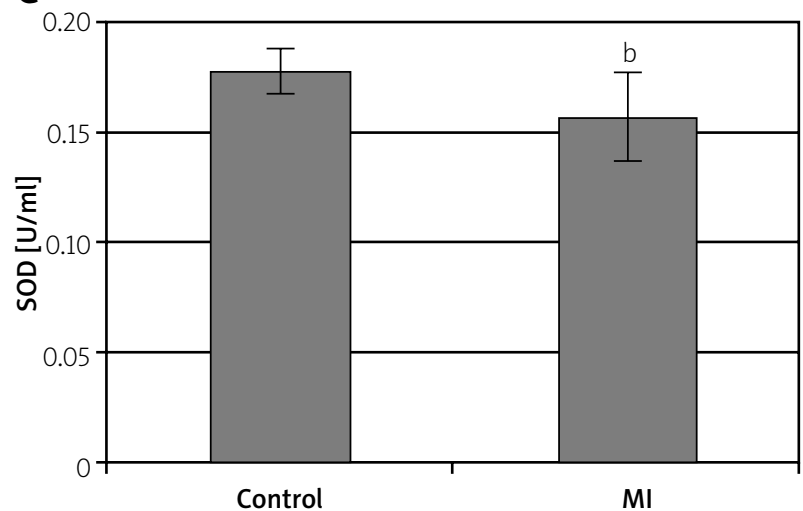

B

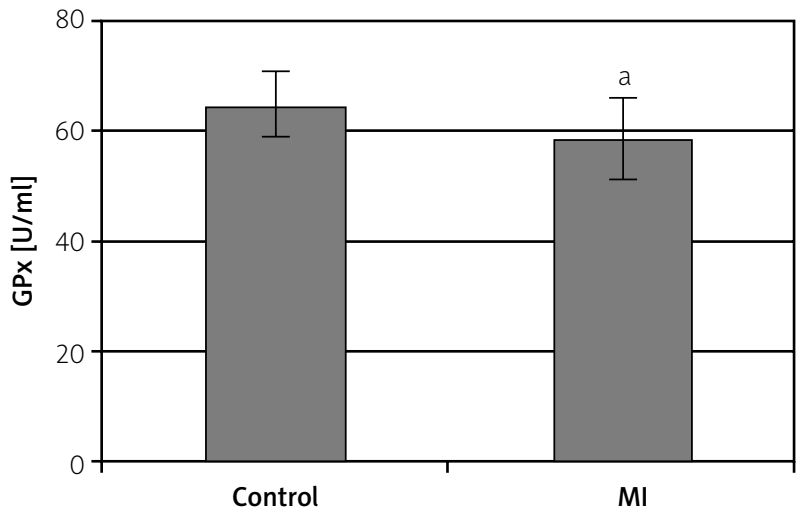

Figure 2. Antioxidant enzyme activities for each group. A - Erythrocyte CAT activity. B - Erythrocyte GPx activity. $\mathbf{C}$ - Erythrocyte SOD activity

${ }^{a} p<0.01$ with respect to control; ${ }^{b} p<0.001$ with respect to control. CAT - Catalase, SOD - superoxide dismutase, GPX - glutathione peroxidase. 
A

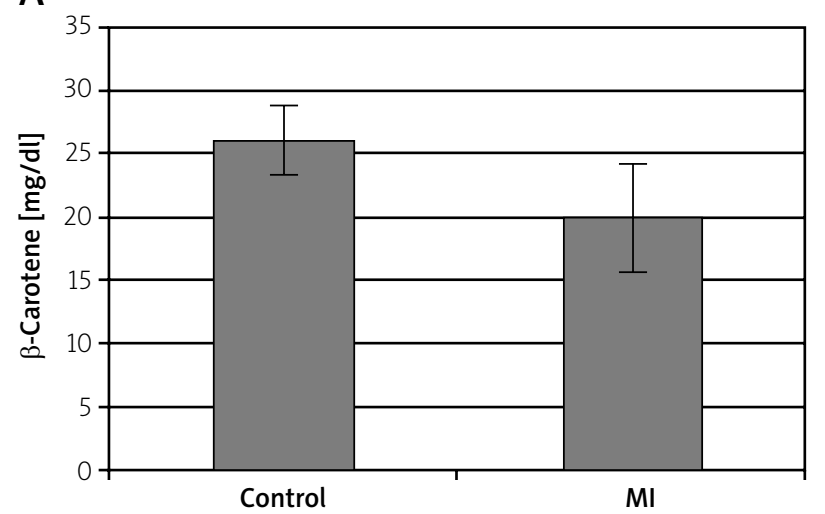

C

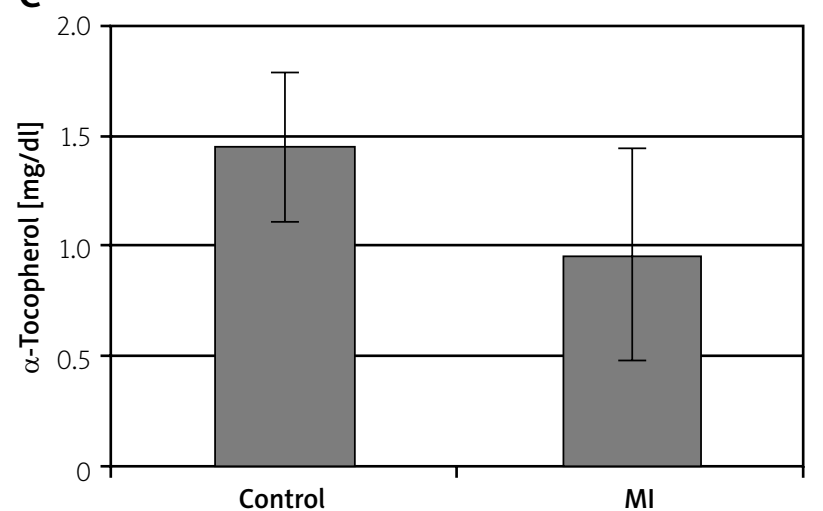

B

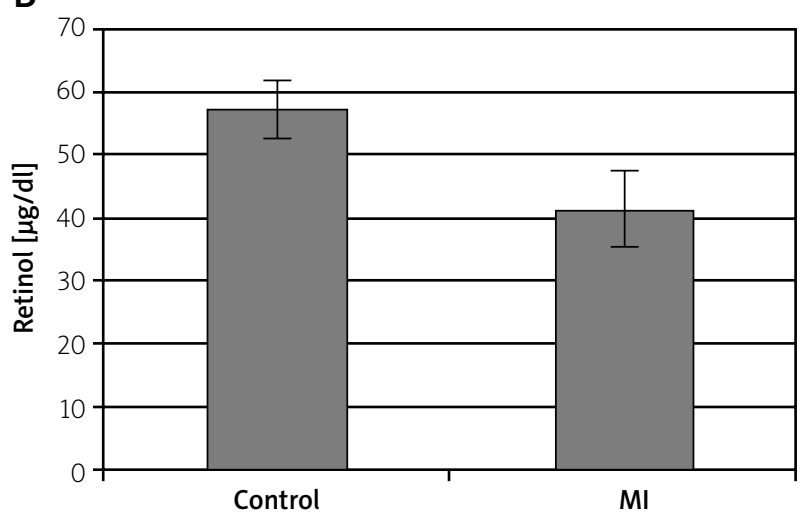

D

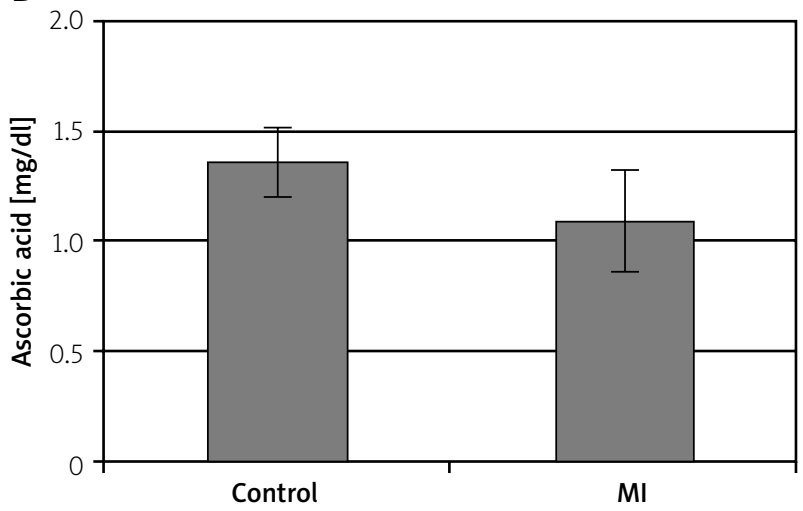

Figure 3. Non-enzymatic antioxidant levels for each group. A - Serum $\beta$-carotene levels. B - Serum retinol levels. C - Serum $\alpha$-tocopherol levels. D - Serum ascorbic acid levels $p<0.05$ with respect to control.

$\pm 0.46)(p<0.001)$, whole blood GSH control group levels (54.054 \pm 5.04$)$ were significantly higher than AMI patient group levels (49.744 \pm 5.17$)(p<0.001)$ (Figure 2).

According to the results of serum ascorbic acid, $\alpha$-tocopherol, retinol and $\beta$-carotene levels, it was observed that non-enzymatic antioxidant levels in the patient group $(1.095 \pm 0.23,0.958 \pm 0.48,41.263 \pm 6.09$ and 19.894 $\pm 4.25)$ were lower than in the control group (1.362 \pm 0.16 , $1.449 \pm 0.34,57.385 \pm 4.49$ and $25.996 \pm 2.75$, respectively). Between groups a statistically significant difference $(p<0.05)$ was determined (Figure 3).

\section{Discussion}

Atherosclerosis takes place in more than half of the deaths in the western world. Coronary atherosclerosis leads to ischemic heart disease (IHD), which mostly has serious consequences [2]. Atherosclerosis, which is one of the most important causes of myocardial infarction, is a kind of vessel disease, characterized by loss of elasticity and thickness in arteries, especially the aorta, coronary and cerebral arteries. Many hypotheses about the causes of atherosclerosis have been proposed. In the light of research it has been demonstrated that the most consistent one is the oxidative stress hypothesis [3]. The relationship between atherosclerosis and oxidative stress is under investigation with experimental studies conducted on animals and humans, by examining the antioxidant and free radical balance in tissues and atheroma [4].

In the present study, between AMI diagnosed patients and control groups, statistically significant changes were also observed for IMA, CK, troponin, MDA, as well as antioxidant enzymes (SOD, GPx, CAT), and non-enzymatic (GSH, $\beta$-carotene, vitamin $\mathrm{A}, \mathrm{C}$ and $\mathrm{E}$ ) antioxidants.

In recent years, for diagnosis of myocardial ischemia, a new parameter has been researched, which is called ischemia modified albumin (IMA), thought to be more advantageous than common methods. In our study, IMA levels were found to be significantly higher in the patient group compared to the healthy group. Santgita et al. and Lin et al. state that CTnI and CK-MB levels increase in heart disease patients. In our study, both markers were found to be very high in patients compared to the control group [21].

Another important parameter related to oxidative stress, occurring as a result of lipid peroxidation caused by ROS activity, is malondialdehyde (MDA) [22]. Pucheu et al. showed that MDA during ischemic myocardial reperfusion was a good oxidative stress indicator [23]. Cannon also remarked that the cause of an increased level of MDA in $\mathrm{AMI}$ patients is cellular damage induced by free oxygen 
radicals [24]. We believe that intense oxidative stress induces the increase of MDA. In addition, MDA plays role in the formation of atherogenesis, which as a result causes ischemic heart diseases. These findings indicate that MDA is an important cardiovascular parameter, which should be followed before and during the disease.

Antioxidants inhibit cell damage related to oxidation, by preventing the formation of active oxygen or by binding to formed active oxygen [25], and are an important part of prevention of tissue damage caused by oxidation [26]. The most important intracellular enzyme that detoxifies free radicals is glutathione peroxidase (GPX) [27]. West et al. showed that SOD and CAT antioxidant enzyme activities decreased in samples collected from a human coronary artery [28]. Another study showed that these parameters were lower in the patient group than the control group [29]. According to Espinola-Klein et al., erythrocyte GPx activity depending on the severity of atherosclerosis showed a decrease with increased cardiovascular risk [30]. In the present study, SOD and CAT levels in patients undergoing $\mathrm{MI}$ were found that decreased significantly as other antioxidant enzymes such as GPx. Activity loss of these enzymes, which are responsible for detoxification of $\mathrm{H}_{2} \mathrm{O}_{2}$ and $\mathrm{O}_{2}$, was shown as a result of oxidative stress in myocardial infarction patients. Especially after myocardial reperfusion, reduction of increased $\mathrm{O}_{2}$ radicals to normal levels by antioxidant enzymes is essential. For prevention of tissue damage, maintaining such enzyme levels within normal values is critical [28-31].

The most important task of glutathione in the organism is reduction of enzyme and protein sulfhydryl groups $(-\mathrm{SH})$ and providing control of reduced forms at sufficient levels [32]. Coppola et al. indicated that administration of GSH in heart patients shows antioxidant effects as well as improving the blood filtration and reducing blood viscosity [33].

Usal et al. stated that erythrocyte GSH levels in the myocardial infarction patient group compared to the control group were significantly higher, and MDA levels were increased [34]. In our study, whole blood GSH control group levels were significantly higher than the $\mathrm{AMI}$ patient group $(p<0.001)$. It is known that reactive oxygen species oxidize thiol groups of proteins. Glutathione which contains - $\mathrm{SH}$ groups in its structure is active in removing the damage. Consequently lower GSH levels in AMI patients were observed.

Ascorbic acid has multiple antioxidant features including the ability to regenerate $\alpha$-tocopherol by reducing $\alpha$-tocopheryl radicals, which are present on the membrane surface [35]. Senthil et al. observed a decrease in vitamin $C$ in cardiogenic shock patients. This decrease could favor the use of vitamin $C$ as an antioxidant defense against increased ROS [29].

Vitamin $\mathrm{E}$ has very strong antioxidant properties, and for this reason several ischemia reperfusion studies have been performed [36]. This non-enzymatic antioxidant plays a role in preventing cell membranes' integrity by limiting lipid peroxidation caused by ROS. $\beta$-Carotene is a unique lipid-soluble chain-breaking antioxidant, which takes the peroxyl radicals [37].

In this study, mean levels of ascorbic acid, retinol, $\alpha$-tocopherol and $\beta$-carotene were significantly lower in AMI patients than the control group, while MDA was significantly higher in AMI patients. In the AMI patient group we found significantly lower levels of ascorbic acid and $\alpha$-tocopherol when compared to the control group. All these data remarks a balance tendency for the benefit of ROS and harmful damage to the antioxidant system, which is unable to compete with oxidative stress and inflammation [38].

Consequently, decrease in the level of enzymatic and non-enzymatic antioxidants, plays role in the development of AMI. As an alternative to heart disease diagnostic methods, among potential subjects, these values should be evaluated. Alternatively to conventional methods, prevention of development of AMI and sudden death, can be achieved by evaluation of these values in potential heart disease patients. However, still more research must be performed and comparison of other factors is necessary in order to be conclusive. In addition, this study indicates that high levels of IMA can be used more widely in the diagnosis of AMI together with other biochemical parameters to obtain more reliable diagnostic data.

\section{Acknowledgments}

This research project was supported by the Scientific and Technological Research Council of Turkey (TUBITAK-SBAG-112S166) and Yıldız Technical University research fund (2013-07-04-YL07).

\section{Conflict of interest}

The authors declare no conflict of interest.

\section{References}

1. Go AS, Mozaffarian D, Roger VL, et al. Executive summary: heart disease and stroke statistics -2013 update: a report from the American Heart Association. Circulation 2013; 127: 143-52.

2. Kumar V, Cotran RS, ve Robbins SL. Robbins basic pathology. PA: Saunders, Philadelphia 2003.

3. Madamanchi NR. Oxidative stress and vascular disease. Arterioscler Thromb Vasc Biol 2005; 25: 29-38.

4. Nakazawa H, Genka C, Fujishima M. Pathological aspects of active oxygens free radicals. Japan J Physiol 1996: 46: 15-32.

5. Cui H, Kong Y, Zhang H. Oxidative stress, mitochondrial dysfunction, and aging. J Signal Transduct 2012; 2012: 646354.

6. Whitaker JM. Dr. Whitaker's Complete guide to natural healing, Washington, DC: Regnery Pub 1996.

7. Fearon IM, Faux SP. Oxidative stress and cardiovascular disease: novel tools give (free) radical insight. J Molecul Cellular Cardiol 2009; 47: 372-81.

8. Baublis AJ, Clydesdale FM, Decker EA. Antioxidants in wheatbased breakfast cereals. Cereal Foods World 2000; 45: 71-4. 
9. Zhao W, Diz D, Robbins ME. Oxidative damage pathways in relation to normal tissue injury. Br J Radiol 2007; 80 (Special issue 1): S23-31.

10. Toker A, Aribas A, Yerlikaya FH, Tasyurek E. Serum and saliva levels of ischemia-modified albumin in patients with acute myocardial infarction. J Clin Lab Anal 2013; 27: 99-104.

11. Jain SK, McVie R, Duett J, Herbst JJ. Erythrocyte membrane lipid peroxidase and glycolylated hemoglobin in diabetes. Diabetes 1989; 38: 1539-43.

12. Griffith OW. Determination of glutathione and glutathione disulfide using glutathione reductase and 2-vinylpyridine. Anal Biochem 1980; 106: 207-12.

13. Suzuki I, Katoh N. A simple and cheap method for measuring serum vitamin A in cattle using spectrophototmeter. Jpn J Vet Sci 1990; 52: 1281-3.

14. Martinek R. Method for determination of vitamin $E$ (total tocopherol) in serum. Clin Chem 1964; 10: 1078-86.

15. Omaye ST, Turnbul JD, Savberlich HE. Ascorbic acid analysis II. Determination after derivatisation with 2.2-dinitrophenylhidrazine. Selected methods for determination of ascorbic acid in animal cells tissues and fluids. In: Methods in enzymology. McCormick DB, Wright LD (eds). Academic Press, New York, USA 1979; 62: 7-8.

16. Suzuki I, Katoh N. A simple and cheap method for measuring serum vitamin A in cattle using spectrophototmeter. Jpn J Vet Sci 1990; 52: 1281-3.

17. Paglia DE, Valentine WN. Studies on the quantitative and qualitative characterization of erythrocyte glutathione-peroxidase. J Lab Clin Med 1967; 70: 158-69.

18. Johansson LH, Borg LA. A spectrophotometric method for determination of catalase activity in small tissue samples. Anal Biochem 1988; 174: 331-6.

19. Sun Y, Oberley LW, Li Y. A simple method for clinical assay of superoxide-dismutase. Clin Chem 1988; 34: 497-500.

20. Bar-Or D, Lau E, Winkler JV. A novel assay for cobalt-albumin binding and its potential as a marker for myocardial ischemia a preliminary report. J Emerg Med 2000; 19: 311-5.

21. Patil SM, Banker MP, Padalkar RK, et al. The clinical assessment of ischaemia modified albumin and troponin I in the early diag nosis of the acute coronary syndrome. J Clin Diagn Res 2013; 7: 804-8.

22. Lin S, Yokoyama H, Rac VE, Brooks SC. Novel biomarkers in diagnosing cardiac ischemia in the emergency department: a systematic review. Resuscitation 2012; 83: 684-91.

23. Pucheu S, Coudray C, Vanzetto G, et al. Assessment of radical activity during the acute-phase of myocardial-infarction following fibrinolysis - utility of assaying plasma malondialdehyde. Free Radical Biol Med 1995; 19: 873-81.

24. Cannon RO. Mechanisms, management and future directions for reperfusion injury after acute myocardial infarction. Nature Clin Pract Cardiovasc Med 2005; 2: 88-94.

25. Baublis AJ, Clydesdale FM, Decker EA. Antioxidants in wheatbased breakfast cereals. Cereal Foods World 2000; 45: 71-4.

26. Subramanian M, Sreejayan Rao MNA, Devasagayam TPA, Singh BB. Diminution of singlet oxygen-induced DNA-damage by curcumin and related antioxidants. Mutat Res 1994; 311: 249-55.

27. Takeshita S, Inoue N, Ueyama T, et al. Shear stress enhances glutathione peroxidase expression in endothelial cells. Biochem Biophys Res Commun 2000; 273: 66-71.

28. West N, Guzik T, Black E, Channon K. Enhanced superoxide production in experimental venous bypass graft intimal hyper- plasia: role of $\mathrm{NAD}(\mathrm{P}) \mathrm{H}$ oxidase. Arterioscler Thromb Vasc Biol 2001; 21: 189-94.

29. Senthil SS, Veerappan R, Rao MR, Pugalendi KV. Oxidative stress and antioxidants in patients with cardiogenic shock complicating acute myocardial infarction. Clin Chim Acta 2004; 348: 131-7.

30. Espinola-Klein C, Rupprecht HJ, Bickel C, et al. Glutathione peroxidase-1 activity, atherosclerotic burden, and cardiovascular prognosis. Am J Cardiol 2007; 99: 808-12.

31. Lobo V, Patil A, Phatak A, Chandra N. Free radicals, antioxidants and functional foods: impact on human health. Pharmacogn Rev 2010; 4: 118-26.

32. Blankenberg S, Rupprecht HJ, Bickel C, et al. Glutathione peroxidase 1 activity and cardiovascular events in patients with coronary artery disease. N Engl J Med 2003; 349: 1605-13.

33. Coppola L, Grassia A, Giunta R, et al. Glutathıne (Gsh) improved hemostatic and hemorheological parameters in atherosclerotic subjects. Drugs Exp Clin Res 1992; 18: 493-8.

34. Acarturk UA, Yuregir E, Unlukurt GT, et al. Decreased glutathione levels in acute myocardial infarction. Japan Heart J 1996; 37 : $177 Z 182$.

35. Padayatty SJ, Katz A, Wang Y, et al. Vitamin C as an antioxidant: evaluation of its role in disease prevention. J Am Coll Nutr 2003; 22: $18-35$.

36. Canbaz S, Duran E, Ege T, et al. The effect of intracoronary administration of vitamin $E$ on myocardial ischemia-reperfusion injury during coronary artery surgery. Thorac Cardiovasc Surg 2003; 51: 57-61.

37. Mueller L, Boehm V. Antioxidant activity of beta-carotene compounds in different in Vitro Assays. Molecules 2011; 16: 1055-69.

38. Patil N, Chavan V, Karnik ND. Antioxidant status in patients with acute myocardial infarction. Indian J Clin Biochem 2007; 22: 45-51. 